\section{A) Check for updates}

Cite this: Nanoscale, 2020, 12, 2292

\title{
Probing the frictional properties of soft materials at the nanoscale
}

\author{
Evangelos Liamas, (D) a Simon D. Connell, ${ }^{b}$ Shivaprakash N. Ramakrishna (D) ${ }^{c}$ and \\ Anwesha Sarkar (iD *a
}

The understanding of friction in soft materials is of increasing importance due to the demands of industries such as healthcare, biomedical, food and personal care, the incorporation of soft materials into technology, and in the study of interacting biological interfaces. Many of these processes occur at the nanoscale, but even at micrometer length scales there are fundamental aspects of tribology that remain poorly understood. With the advent of Friction Force Microscopy (FFM), there have been many fundamental insights into tribological phenomena at the atomic scale, such as 'stick-slip' and 'super-lubricity'. This review examines the growing field of soft tribology, the experimental aspects of FFM and its underlying theory. Moving to the nanoscale changes the contact mechanics which govern adhesive forces, which in turn play a pivotal role in friction, along with the deformation of the soft interface and dissipative phenomena. We examine recent progress and future prospects in soft nanotribology.

Received 16th August 2019, Accepted 2nd December 2019 DOI: $10.1039 / c 9 n r 07084 b$ rsc.li/nanoscale

\section{Introduction}

Friction is the force resisting relative motion between two sliding bodies, spanning many orders of magnitude in length, time and energy scales. It has far-reaching implications in science and engineering, from carbon nanotube bearings ${ }^{1}$ to biological lubrication ${ }^{2-4}$ and geophysical faults ${ }^{5}$ in earthquakes, placing it at the forefront of current research. Friction becomes particularly relevant at the nanoscale in a wide range

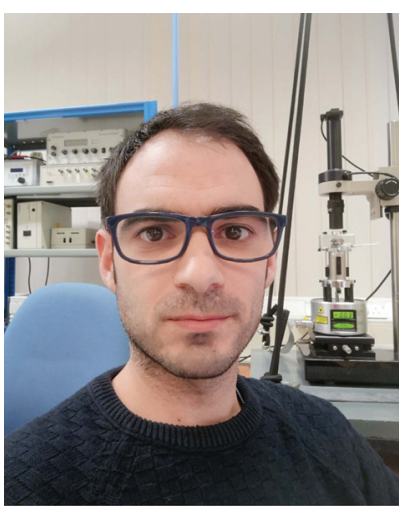

Evangelos Liamas
Dr Evangelos Liamas completed his PhD at the Department of Chemical Engineering at the University of Birmingham, UK, where he studied interactions between proteins and biomaterials and the impact of protein adsorption at sliding interfaces. Since 2018 he has been a postdoctoral research fellow at the University of Leeds, UK, working on a European Research Council (ERC) funded project - LubSat. His research focusses on measuring frictional forces on soft biological tissues and biomimetic polymers, in order to achieve a fundamental understanding of biolubrication at the nanoscale.

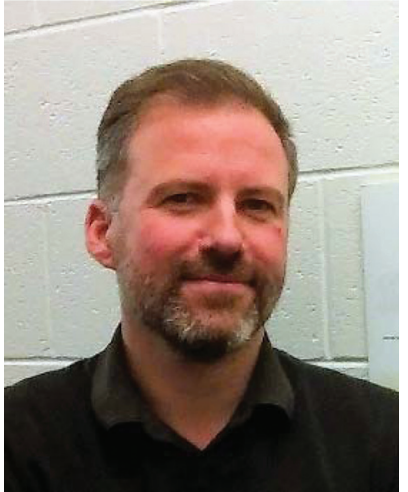

Simon D. Connell
Dr Simon Connell is an Associate Professor at the School of Physics and Astronomy, has two decades of experience in atomic force microscopy, and currently manages a cross-faculty AFM facility. He studies a wide range of systems, with a focus on the structure, dynamics and nanomechanics of soft matter and biological systems, largely in a fully hydrated state. Particular areas of interest are biomembrane phase behaviour, blood clot structure and mechanics, hydrogels and microgels, and high speed imaging of polymer dynamics. 
of systems operating under mild to extreme load conditions. Applications of nanoscale friction spanning from device miniaturization i.e. micro- and nano-electromechanical devices (MEMS/NEMS $)^{6}$ to complex frictional phenomena occurring in biological applications including protein motors on microtubules $^{7}$ to prokaryotic/eukaryotic cells under naturally-occurring sliding motions ${ }^{8}$ and biomimetics ${ }^{9}$ have placed it at the cutting edge of nanotechnological research. Over the past three decades, friction force microscopy (FFM) based on atomic force microscopy (AFM) has emerged as a central nanomechanical tool, which has made possible the accurate quantification of lateral forces between the tip of a microfabricated cantilever and the samples with a sensitivity of a few piconewtons. ${ }^{10}$

Due to the exciting advances in FFM enabling tremendous progress in decoding complex physical, biological and technological lubrication mechanisms at the nanoscale, there have been some excellent reviews. ${ }^{11-15}$ Many, if not most, of these reviews focus on summarizing particular contact mechanics at the nanoscale in conventional 'hard' interfaces with a welldefined single-asperity elastic contact. However, there are no reviews that discuss nanotribology in deformable i.e. low modulus 'soft' surfaces, such as polymers, hydrogels, and soft biological interfaces (oral, ocular, dermal, and respiratory), which is one of the outstanding challenges in modern nanotribology. In this Review, we specifically provide a critical analysis of FFM focusing on the booming area of nanotribology in soft surfaces, describing the rapid evolution of FFM, the gradual transition from the use of sharp tips ${ }^{16}$ to well-defined colloidal probes, ${ }^{17}$ and more recently performing friction measurements incorporating flexibility into the material physics and chemistry of the contact surfaces. ${ }^{18}$ We explore the frictional laws applying to soft surfaces at the nanoscale, highlighting the effect of adhesion force on the frictional behaviour of soft surfaces, which is an outstanding challenge in modern tribology. We extensively review studies where FFM has been used on soft surfaces to measure these tiny lateral forces, and discuss the impact that (i) surface interactions, (ii) surface roughness, (iii) intrinsic material properties, and (iv) experimental conditions have on the frictional properties of hard-onsoft and soft-on-soft contact systems. We examine aspects of molecular dynamics (MD) simulations that allow the prediction of frictional behaviour at the nanoscale for hard and soft contacts. Lubrication on soft surfaces is a complex multidisciplinary area, and we touch upon the key surface interactions altered by grafting polymer brushes and hydrogels. Readers might refer to more extensive reviews on polymer brushes, hydrogels and hydration lubrication elsewhere. ${ }^{19-21}$ Finally, we outline the systems where FFM using soft surfaces can be applied, before looking into the future opportunities, including the fabrication of precisely tailored soft probes for FFM along with the growing need for new mathematical models to overcome the current limitations of FFM-based approaches for soft tribology.

\section{Evolution of friction force measurements at the nanoscale}

The atomic force microscope was invented in 1986 by Binnig et $a l .,{ }^{22}$ and was capable of tracking a surface with a $1 \AA$ vertical resolution. Very shortly after, motivated by the knowledge gap in the atomic dynamics of friction forces, Mate et al. ${ }^{16}$ modified the AFM to enable detection of the cantilever lateral deflection using optical interference, enabling the detection of friction forces for the first time (Fig. 1). They used a sharp tungsten wire, sliding over a graphite surface with a wide range of velocities under varying normal loads, observing

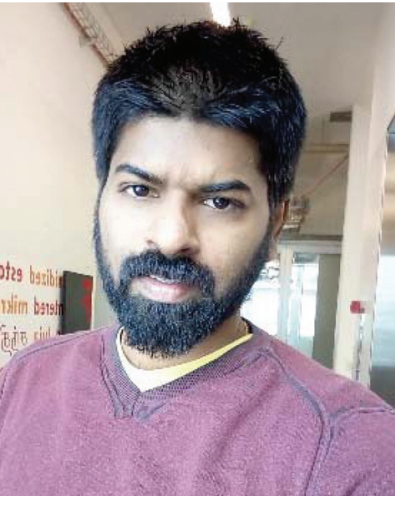

Shivaprakash N.

Ramakrishna
Dr Shivaprakash N. Ramakrishna completed his Bachelor's and Master's degrees at Kuvempu University, Karnataka, India. He carried out his PhD degree in 2013 at the Department of Materials, ETH Zurich, Switzerland under the guidance of Prof. Nicholas D. Spencer. He worked as a postdoctoral researcher at the Laboratory for Surface Science and Technology from 2013 to 2015. Since 2015, he has been a senior scientist in the same laboratory, focusing his research on surface science, nanotribology, nanomechanics and soft matter.

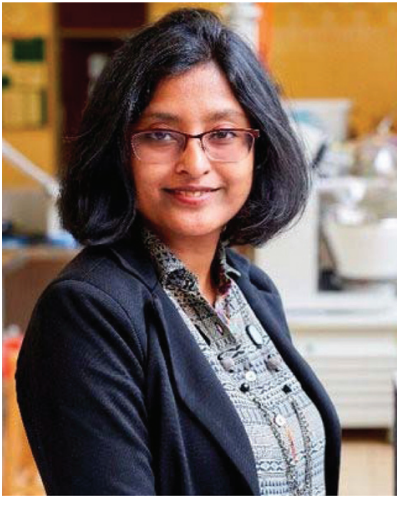

Anwesha Sarkar
Dr Anwesha Sarkar is an Associate Professor of Food Colloids at the School of Food Science and Nutrition at the University of Leeds, UK, where she leads a European Research Council (ERC) funded project LubSat on unravelling the fundamental mechanisms behind oral lubrication at multiple length scales. Her research focuses on the tribological properties of soft surfaces for applications in food, healthcare, and allied soft materials. She develops novel bioinspired surfaces at macro to nanoscale appropriate for studying the lubrication properties of protein films, self-assemblies, microgels, emulsions and other colloidal systems. To date, she has published 75 peer-reviewed articles and book chapters and is the co-inventor of 5 patents. 

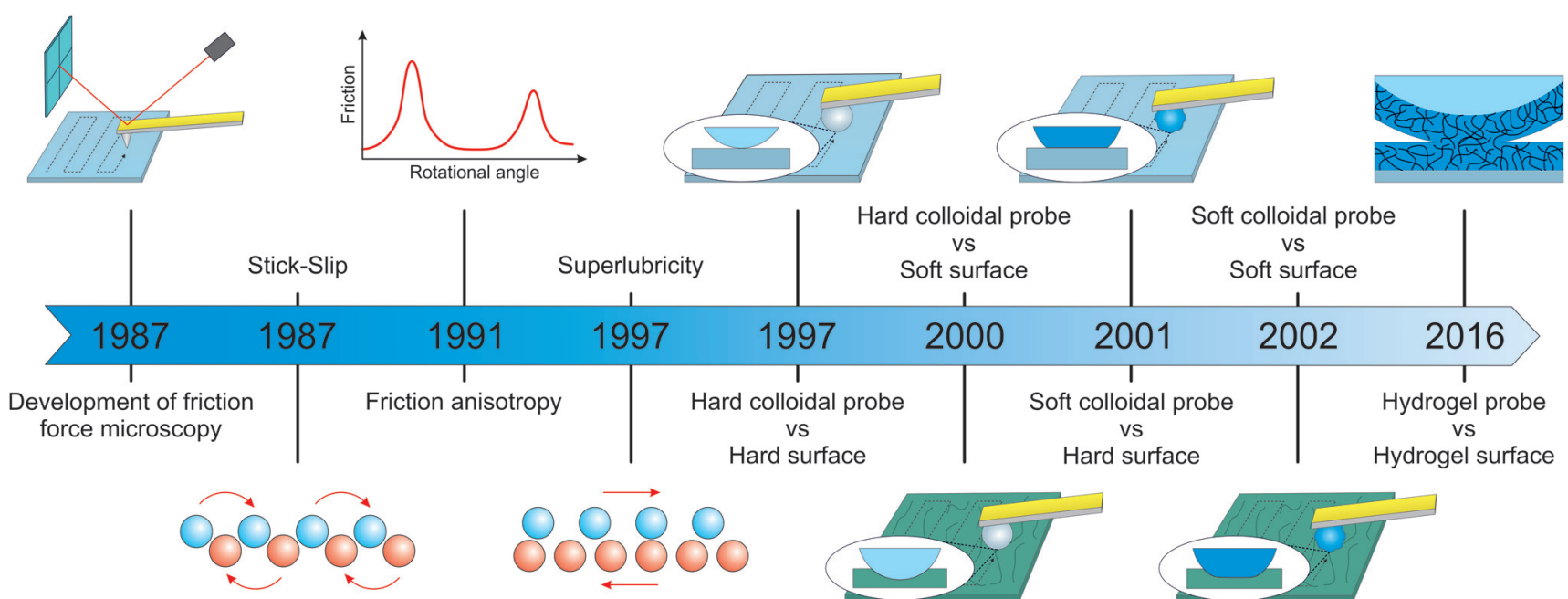

ard colloidal probe vs

Soft surface

Fig. 1 Evolution of friction force microscopy (FFM) with major milestones leading to its use in soft surfaces. Development of FFM dates back to Mate et al. ${ }^{16}$ who used a tungsten tip against a graphite surface. They were the first to observe a 'stick-slip' phenomenon, which occurs when the tip sticks to one lattice site of the sample until the lateral force becomes strong enough to jump to the next site. Friction 'anisotropy' and 'superlubricity' were firstly observed by Hirano et al. ${ }^{24}$ and Overney et al., ${ }^{23}$ respectively, although this phenomenon was demonstrated elegantly later by Dienwiebel et al. ${ }^{29}$ by measuring lateral forces as a function of rotational angle. In the case of 'anisotropy', friction between two crystal surfaces depends on their molecular alignment, where friction is highest when the crystal lattices are commensurate. On the other hand, 'superlubricity' or ultra-low friction occurs when the lattices of two crystal surfaces in contact are out of registry by lattice mismatch or angular misalignment, because the sum of the forces that act on the atoms of each surface cancel each other. Toikka et al. ${ }^{17}$ was the first group to use a colloidal probe to measure lateral force using a hard probe/hard surface system. Subsequently, Matzelle et al. ${ }^{30}$ studied a hard probe/soft surface, while Bogdanovic et al. ${ }^{31}$ used materials other than glass to create a relatively soft cellulose-based colloidal probe. Kim et al. ${ }^{32}$ used a soft colloidal probe/soft surface system for the first time to study friction on hydrogel contact lenses, while Li et al. ${ }^{33}$ used a hydrogel probe/hydrogel surface to understand the lubrication mechanism of crosslinked hydrogel layers.

stick-slip behaviour with a period of $0.25 \mathrm{~nm}$ and that matches the graphite lattice, and a dynamic friction proportional to load indicative of a single point contact. Over the following two decades the main focus was to uncover fundamental interactions at the atomic scale, with further insights into 'stick-slip', ${ }^{16}$ and discovery of 'superlubricity' ${ }^{23}$ and 'anisotropy'24 (Fig. 1). However, in terms of instrumentation, the main advance came with the introduction of colloidal probes of well-defined geometries, that were used instead of sharp tips of unknown or difficult to measure tip radius (Fig. 2). With the use of contact mechanics models, the probesample contact area could now be estimated and hence an absolute quantitative frictional force could be determined. At the same time, while measuring friction with commercial AFM tips is limited to the use of silicon or silicon nitride as a material with elastic modulus $>10 \mathrm{GPa}$, colloidal probes offer a greater variety of choice regarding the material chemistry, ${ }^{25,26}$ elastic modulus $<10 \mathrm{GPa},{ }^{27,28}$ and probe radius of hundreds of nanometers to few micrometers (Fig. 2).

Ducker et $a l .{ }^{34}$ were the first to use a colloidal probe in 1991 to measure normal forces between a silica colloidal particle ( $3.5 \mu \mathrm{m}$ radius) attached to a silicon nitride cantilever and a flat silica surface in sodium chloride solutions. They found general agreement with DLVO theory, with the exception of some unexpectedly high forces at close range $<3 \mathrm{~nm}$. Nevertheless, it was only in 1997 that Toikka et al. ${ }^{17}$ used a colloidal probe to measure lateral forces. Interestingly, this was driven by interest in the force required to remove a single particle of iron oxide from a silica surface.

Since then, colloidal probe FFM has been used to study frictional interactions on a plethora of combinations between hard and soft materials. For instance, Matzelle et al. ${ }^{30}$ studied the tribological properties of soft hydrogel surfaces ( $N$-isopropylacrylamide) for the first time using micrometersized hard glass spheres over a range of loads $(<110 \mathrm{nN})$ for applications in medical devices, such as catheters. The key research challenge of understanding the friction and adhesion occurring at the hydrogel soft contact lens/ocular tissue interface led to the first use of soft-on-soft contact mechanics at the nanoscale i.e. a relatively soft polystyrene colloidal probe (modulus $\approx 3 \mathrm{GPa}$ ) sliding against a hydroxyethyl methacrylate-based hydrogel (modulus ranging $0.5-1 \mathrm{MPa}$ ) by Kim et $a l^{32}$ (Fig. 1). Here, they found that ionic functional groups reduced surface adhesion and friction against a hydrophobic probe. Due to the technical difficulties of attaching an ultra-soft particle (e.g. hydrogel particle) to an AFM cantilever, friction between ultra-soft systems at the nanoscale was not measured until 2016. It was only recently, when Li et al. ${ }^{33}$ were able to perform FFM experiments between a hydrogel probe and hydrogel surface. They used an elegant approach where a polystyrene colloidal probe was coated with a poly(dimethyl acrylamide-co-methacryloyl oxybenzophenone) (PDMAA-co-MABP)-based hydrogel layer to elucidate lubrication on a hydrogel substrate. They reported very low 

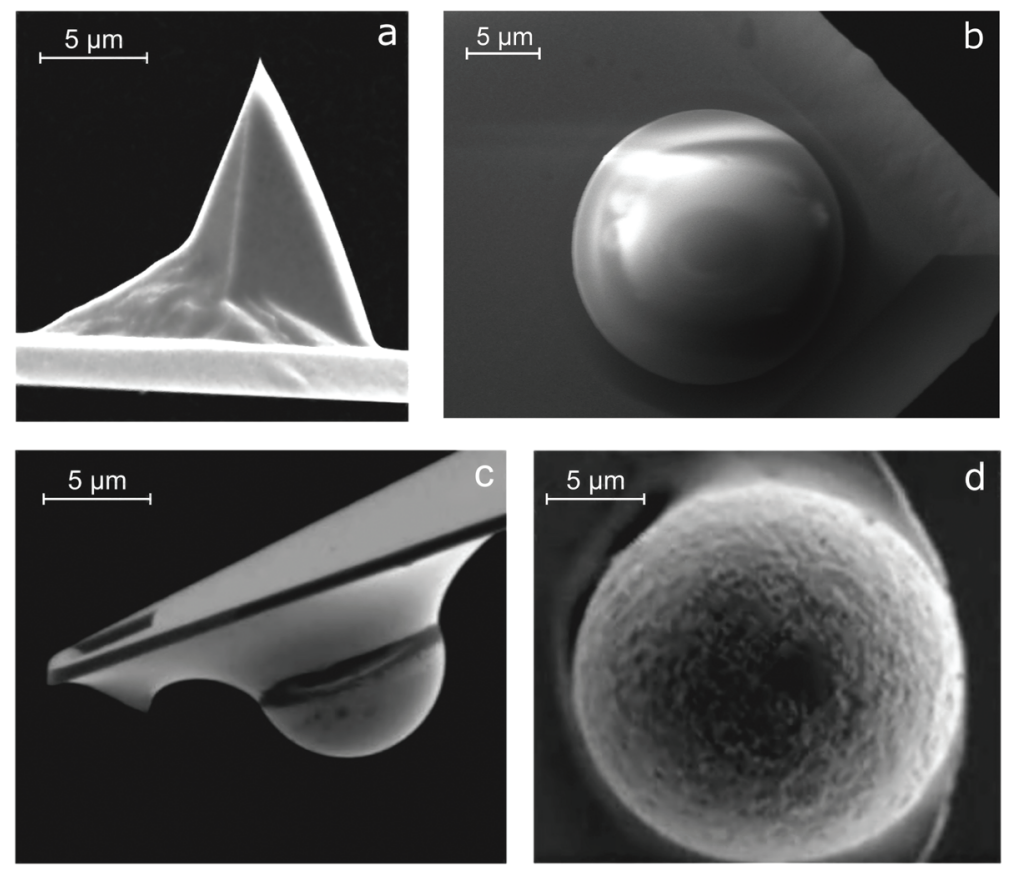

Fig. 2 Scanning electron microscopy images of the sharp tip and colloidal probes glued onto AFM cantilevers. (a) Commercial AFM cantilevers made from silicon or silicon nitride, having a sharp tip with a radius of a few nanometers, are widely being used to measure friction (adapted with permission from J. Sondhauss, M. Lantz, B. Gotsmann and A. Schirmeisen, Langmuir, 2015, 31, 5398-5405. ${ }^{35}$ Copyright 2015 American Chemical Society). Following the introduction of colloidal probes in FFM, several materials have been used to study friction, including (b) polydimethylsiloxane (PDMS) (our laboratory, unpublished work), (c) borosilicate glass (adapted from J. M. Coles, J. J. Blum, G. D. Jay, E. M. Darling, F. Guilak and S. Zauscher, J. Biomech., 2008, 41, 541-548, ${ }^{36}$ Copyright 2008, with permission from Elsevier), and (d) polyethylene (PE) (adapted with permission from S. N. Ramakrishna, P. C. Nalam, L. Y. Clasohm and N. D. Spencer, Langmuir, 2013, 29, 175-182.28 Copyright 2013 American Chemical Society).

friction coefficients $(\mu=0.006)$ between the hydrated hydrogel surfaces, which increase with the sliding speed, and revealed that pressure-induced deswelling and hydration lubrication in the contact region dominates friction. Consequently, measuring friction between the hydrogel-coated probe and hydrogel surface offers new opportunities to understand frictional dissipation in deformable soft biological samples.

\section{Friction laws at the macro and nanoscale}

Friction laws between two macroscopic sliding objects were first described by Leonardo da Vinci ${ }^{37}$ in the $15^{\text {th }}$ century to be "independent of the apparent area of contact" and "proportional to the load pressing the surfaces together", which was formulated 200 years later by Guillaume Amontons, as expressed in eqn (1):

$$
F_{\mathrm{f}}=\mu L
$$

where, $F_{\mathrm{f}}$ is the frictional force, $\mu$ is the friction coefficient, and $L$ is the normal load. ${ }^{38}$ The phenomenological independence of friction from the contact area at the macro- scale arises from the fact that the real contact area between two surfaces is much smaller than the apparent area ( $\left.A_{\text {macro }}\right)$. This has been proven elegantly by Bowden, ${ }^{39}$ through measuring the electrical resistance of metals in contact, who found that the real area of contact can be as small as 1/10 000 of the apparent contact area. He also reported that the real contact area is linearly proportional to the applied load and approximately independent of the surface size. Based on this, Bowden and Tabor $^{40}$ explained the difference in the dependence of friction on the contact area at the macro and nanoscale as follows; at the macroscale, the real contact area $\left(\sum A_{\text {asp }}\right)$ is the summation of a large number of smaller contacts (asperities, $A_{\text {asp }}$ ) which is much smaller than $A_{\text {macro }}$ (Fig. 3a), and the independence of friction from $A_{\text {macro }}$ is due to the increase of $A_{\text {asp }}$ with increasing load caused by asperity deformation, balancing the contact pressure, whereas at the nanoscale, $A_{\text {asp }}$ is directly proportional to the friction force.

Considering that friction is determined by factors such as plastic deformation, ${ }^{43}$ wear, ${ }^{44}$ surface roughness, ${ }^{28}$ and lubrication, ${ }^{45}$ creating a universal law that describes friction across all length scales from macro down to nano is a major challenge. With the development of FFM, it became possible to control all the aforementioned factors and study friction at the nanoscale, where single asperity interactions dominate and 

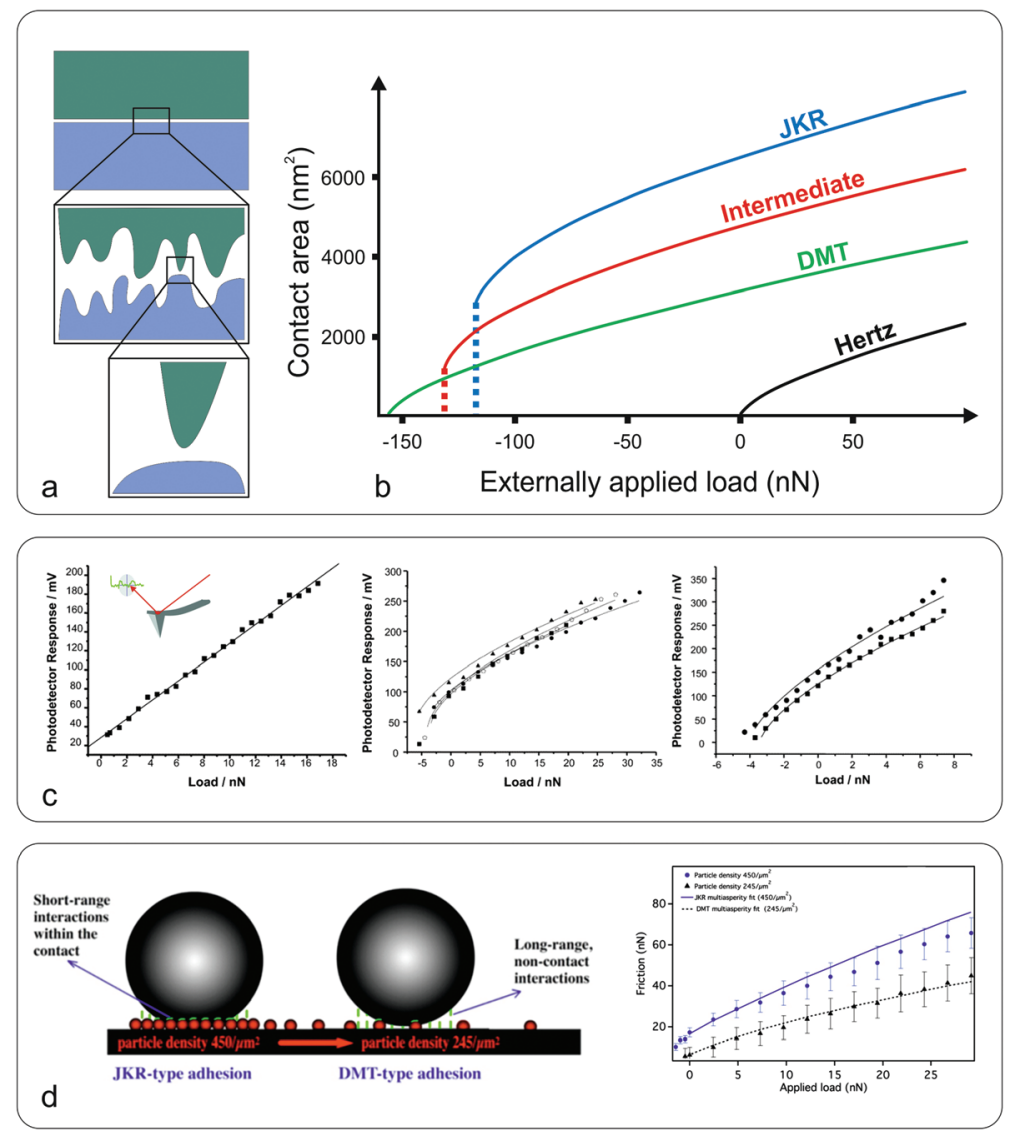

Fig. 3 Macro to nanoscale contact area and contact mechanics models. (a) While at the macroscale the contact area may appear flat (top), at the microscale the surface can be a rough, multi-asperity interface (middle) that consists of nanoscale asperities (bottom). (b) Dependence of the contact area on the applied load, between a sphere and a flat plane, for the Hertz, JKR, DMT, and intermediate models. The calculations (adapted with permission from J. Y. Park and M. Salmeron, Chem. Rev., 2014, 114, 677-711 ${ }^{41}$ Copyright 2014 American Chemical Society) were performed for radius $R=100 \mathrm{~nm}$, reduced modulus $K=50 \mathrm{GPa}$, interfacial energy $\gamma=250 \mathrm{~mJ} \mathrm{~m}{ }^{-2}$, and equilibrium separation distance $z_{0}=3 \AA$. (c) Frictional response versus applied load between a silicon nitride sharp tip (inset) and poly(ethylene terephthalate) (PET) surfaces under an ethanol (left), perfluorodecalin (middle), and hexadecane (right) environment (adapted with permission from C. R. Hurley and G. J. Leggett, Langmuir, 2006, 22, 4179-4183. ${ }^{42}$ Copyright 2006 American Chemical Society). The adhesion varies upon the type of solvent due to the different dielectric constants altering adhesive van der Waals forces. Consequently the friction versus load curves range from linear (ethanol) to sub-linear, the latter fitted to DMT (hexadecane) or a JKR model (perfluorodecalin). (d) Schematic representation of the interaction forces acting at the colloidal probe-nanoparticle contact (left), and friction versus load at high (JKR-type adhesion) and low (DMT-type adhesion) particle density (right) (adapted with permission from S. N. Ramakrishna, P. C. Nalam, L. Y. Clasohm and N. D. Spencer, Langmuir, 2013, 29, 175-182. ${ }^{28}$ Copyright 2013 American Chemical Society).

friction is proportional to the normal load, the real contact area, and in many cases to the sliding speed. ${ }^{41}$

The contact area $(A)$ between an AFM tip and a flat surface, when the load is limited to cause only elastic deformation, can be given by the Hertzian model ${ }^{46}$ (eqn (2)):

$$
A=\pi\left(\frac{R L}{K}\right)^{2 / 3}
$$

where $R$ is the tip radius, $L$ is the applied load, and $K$ is the reduced Young's modulus given by eqn (3):

$$
\frac{1}{K}=\frac{3}{4}\left(\frac{1-v_{\mathrm{s}}^{2}}{E_{\mathrm{s}}}+\frac{1-v_{\mathrm{t}}^{2}}{E_{\mathrm{t}}}\right)
$$

where $E_{\mathrm{s}}$ and $E_{\mathrm{t}}$ are the Young's moduli and $v_{\mathrm{s}}$ and $v_{\mathrm{t}}$ are the Poisson's ratios of the surface and the tip, respectively.

However, the Hertz model does not take into consideration the adhesion between the contact surfaces, which plays a major role in friction since it affects the contact area between the tip and the surface. Generally, when adhesion between the tip and surface is small or zero, a linear Amontons' type relationship between friction and load is observed at the nanoscale. ${ }^{42,46}$ In contrast, when the adhesion is significant at the contact, the friction-load follows a sublinear relation that is described well by single asperity contact mechanics models such as the JohnsonKendall-Roberts $(\mathrm{JKR})^{48}$ and the Derjaguin-MullerToporov $(\mathrm{DMT})^{49}$. Such behaviour is often explained by the dependence of the friction force $\left(F_{\mathrm{f}}\right)$ on two terms, a 
load-dependent term and an interfacial shear term ${ }^{46,47}$ (eqn (4)):

$$
F_{\mathrm{f}}=\mu L+\sigma A_{\mathrm{asp}}
$$

where $\sigma$ is the interfacial shear strength. When the adhesion is negligible, the shear term (2nd term in eqn (4)) is insignificant and a linear relationship between friction and load is observed. However, when adhesion is large, the shear term dominates and friction force follows a sublinear relation with the applied load. ${ }^{50}$ Therefore, for adhesive surfaces the JKR and the DMT models are more appropriate for the calculation of the contact area, which are given by eqn (5) and (6), respectively:

$$
\begin{gathered}
A=\pi\left\{\frac{R}{K}\left[L+3 \pi R \gamma+\left(6 \pi R \gamma L+(3 \pi R \gamma)^{2}\right)^{1 / 2}\right]\right\}^{2 / 3}(\mathrm{JKR}) \\
A=\pi\left[\frac{R}{K}(L+2 \pi R \gamma)\right]^{2 / 3}(\mathrm{DMT})
\end{gathered}
$$

where $\gamma$ is the work of adhesion that is given by $\gamma=2 L_{\mathrm{ad}} / 3 \pi R$ and $\gamma=L_{\mathrm{ad}} / 2 \pi R$ for JKR and DMT ( $L_{\mathrm{ad}}$ being the negative adhesive load), respectively. The JKR model calculates the contact area by taking into consideration short-range interactions within the contact area and assuming that no forces act outside the area of contact, while the DMT model considers only the long-range non-contact interactions outside the contact area. Consequently, JKR is a more appropriate model to use when performing FFM on soft materials having high surface energy and a tip or colloidal probe with a large radius, while the DMT model is more suited to describe less deformable materials with low surface energy and a small tip radius. In order to apply the Hertz, JKR, and DMT models there are several assumptions to be met: ${ }^{51}$

- the materials are elastically isotropic;

- the deformation in the contact is purely elastic and is described by classical continuum elasticity theory;

- the diameter of the tip is significantly larger than the diameter of the contact area;

- the Young's modulus and the Poisson's ratio remain constant during deformation;

- no chemical bonds are formed during adhesion;

- the curvature of the particle in the contact area is described by a paraboloid; and

- the contact area is significantly larger than the atomic/ molecular dimensions.

A nice illustration on the relation between contact area and load, using the different models, was given by Park and Salmeron $^{41}$ (Fig. 3b). They showed that under the DMT and JKR models, a finite contact area exists even at zero applied load between the tip and the surface, which arises from the adhesion. Many studies have successfully used friction models that take into account the adhesive contribution according to DMT/JKR. For instance, it has been shown that friction between an AFM tip and poly(ethylene terephthalate) (PET) surfaces is reduced in the presence of ethanol as a result of a reduction in adhesion, leading to a linear friction-load dependence ${ }^{42}$ (Fig. 3c). However, in perfluor- odecalin and hexadecane that have significantly smaller dielectric constants and refractive indices, the adhesion was remarkably larger as a result of stronger dispersion forces according to the Lifshitz theory of van der Waals forces. ${ }^{52}$ Consequently, in perfluorodecalin, where the adhesion was the largest, the frictionload was best described by JKR, while for hexadecane that had a smaller adhesion compared to perfluorodecalin the DMT model was the best fit. The JKR-DMT transition was shown elegantly in a study by Ramakrishna et al., ${ }^{28}$ where the roughness of a surface was controlled with nanoparticles, and then correlated to the adhesion forces and to their frictional properties (Fig. 3d). Friction reduced as particle density decreased (although it sharply increased once the colloidal probe made contact with the underlying flat substrate), confirming that the friction-load relationship is governed by the real contact area, such as asperities on real surfaces. It was shown that JKR contact adhesion dominated the frictional response at high particle density, where many particles came into contact with the colloidal probe. However, when the particle density is reduced, a transition to DMT contact mechanics is observed and adhesion is dominated by long range noncontact forces from the underlying substrate, nanometres distant.

The most appropriate contact model to use in a given system can be determined using the dimensionless Tabor parameter $^{53}(\tau)$ which takes account of the ratio of elastic deformation to the range of adhesive forces, i.e. the balance between JKR and DMT respectively, given by eqn (7):

$$
\tau=\sqrt[3]{\frac{16 R \gamma^{2}}{9 K^{2} z_{0}^{3}}}
$$

where $z_{0}$ is the equilibrium separation distance. For $\tau \leq 0.1$, the contact area is best described using the DMT model, while for $\tau \geq 5$ the JKR model is more appropriate. However, many, if not most, applications fall in the intermediate region between these two extremes. In this region, the Maugis-Dugdale model can be used due to its high degree of flexibility. ${ }^{54}$ Here, Maugis used a Dugdale (square well) model to analyse the JKR and DMT models and suggested that the transition between the two can be predicted from a dimensional parameter $\lambda^{54}$ that is roughly equal to the Tabor parameter and is given by eqn (8):

$$
\lambda=\frac{2.06}{z_{0}} \sqrt[3]{\frac{R \gamma^{2}}{\pi K^{2}}}
$$

However, the Maugis-Dugdale model requires a complicated fitting in the absence of a single parameter relating contact area and normal load. In 1999 Carpick et al. ${ }^{55}$ proposed a general transition equation (GTE) that provides a very close approximation of the Maugis-Dugdale model and is more convenient to use for fitting data from FFM experiments given by eqn (9):

$$
\frac{a}{a_{0(\alpha)}}=\left(\frac{\alpha+\sqrt{1-L / L_{\mathrm{c}(\alpha)}}}{1+\alpha}\right)^{2 / 3}
$$

where $a$ is the contact radius, $a_{0}$ is the contact radius at zero normal load, $\alpha$ is the transition parameter, and $L_{\mathrm{c}}$ is the criti- 
cal load. The two extremes of the transition parameter, $\alpha=1$ and $\alpha=0$, correspond to the JKR and DMT cases, respectively. The GTE is used to fit the experimental data (friction versus load) acquired from FFM, after replacing all occurrences of $a$ in the eqn (9) with $\sqrt{F_{\mathrm{f}}}$ since $F_{\mathrm{f}}=\tau^{*} \pi a^{2}$ where $\tau$ is the constant interfacial shear strength, leaving $a_{0}, L_{\mathrm{c}}$, and $\alpha$ as free parameters that are extracted from the fit. Subsequently, the work of adhesion and interfacial shear strength, $\sigma$, can also be obtained.

\section{Friction force microscopy on soft surfaces}

The majority of FFM studies have been performed on hard and flat surfaces with the focus being on single asperity contact. Interestingly, there has been a gradual shift towards measuring friction force on soft materials, partly attributed to the demands of understanding friction mechanisms at biological interfaces, such as cells ${ }^{3}$ and tissues, ${ }^{36}$ where modulus may range from a few pascals to hundreds of kilopascals. Also, in part, such hierarchically patterned biological interfaces with heterogeneous modulus ${ }^{56}$ can now be fabricated relatively easily thanks to the fascinating parallel development in polymer chemistry and soft lithography, ${ }^{57}$ enabling a more systematic study of friction on soft materials.

Fig. 4 summarizes the literature where FFM has been used to study the frictional properties of soft materials, using either a sharp tip or a colloidal probe. The $y$-axis separates the samples studied according to their Young's moduli, while the $x$-axis indicates the material of the tip, divided into sharp tips to the left of the diagram, or the Young's modulus of the colloidal probe used to the right of the divide. Sharp tips have the benefit that they can be used to study single asperity contacts, and it can be seen that sharp tips have been used against samples with a wide range of moduli, spanning from MPa to GPa. Although they are commercially available and, thus, convenient to use, they are limited to silicon ( $\mathrm{Si}$ ) and silicon nitride $\left(\mathrm{Si}_{3} \mathrm{~N}_{4}\right)$. On the other hand, colloidal probes offer an unlimited palette of materials that can be used, some of which are now commercially available, ranging from borosilicate glass and gold to polystyrene (PS) and poly(methyl methacrylate) (PMMA).

Silica-based colloidal probes are the most widely popular choice to measure friction on materials with Young's modulus in the GPa range, mainly due to their availability and ease of altering the surface interactions using chemical derivatisation. Cellulose has been studied extensively in the work of Rutland et al. ${ }^{31,102,103,105-107,109}$ Polymers, such as PMMA, PS, and polyethylene (PE), are also widely used due to their easily tuneable properties. Although both sharp tips and colloidal probes with elastic modulus $(E)>1 \mathrm{GPa}$ have been used to study friction on surfaces with $E$ down to a few $\mathrm{kPa}$, there is a clear knowledge gap in using colloidal probes and samples that have a Young's modulus of less than $100 \mathrm{MPa}$ (green-shaded area) where most biological interfaces range and soft-on-soft contacts predominate. At the present time, the question is open as to whether a hard-on-soft contact can capture the features of a soft-on-soft contact. We now focus on FFM studies carried out on soft materials (Fig. 5), and more specifically we discuss how surface interactions and topography affect friction in soft contact mechanics, before we examine the impact of intrinsic properties of the material (e.g. polymer entanglement, molecular weight) on friction and other experimental factors, such as loads, scanning distance and sliding speeds.

\subsection{Effect of surface interactions on nanoscale friction}

Surface interactions, of either adhesive or repulsive nature, play a major role in nanoscale frictional properties between sliding objects. It is known $^{41}$ that adhesion between sliding surfaces affects the measured frictional forces in softer

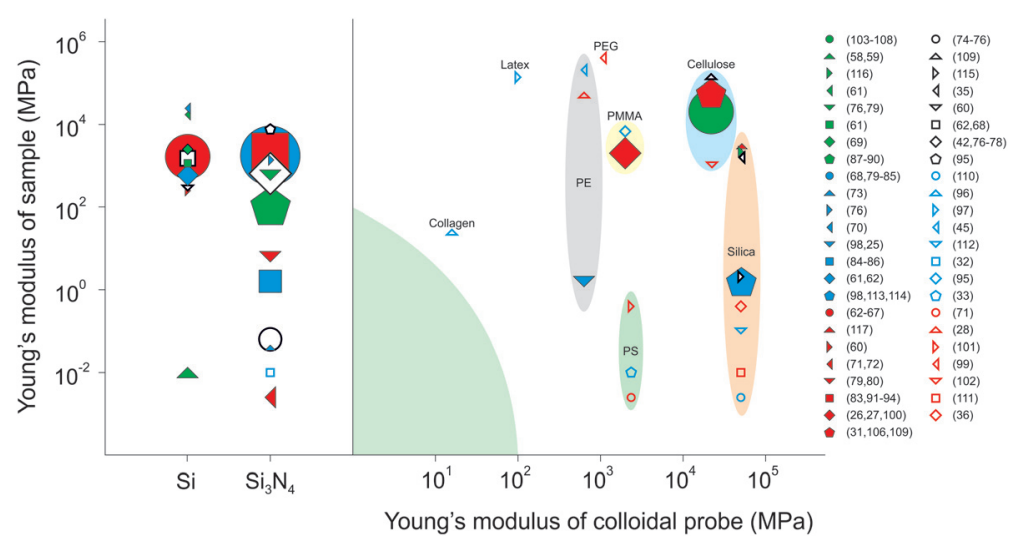

Fig. 4 Material properties of tip/colloidal probes and surfaces used in friction force microscopy (FFM). The $y$-axis separates the samples studied according to Young's modulus, while the $x$-axis indicates the modulus and material of the tip. The left side of the figure illustrates FFM studies using a sharp tip, either silica, ${ }^{58-70}$ or silicon nitride. ${ }^{32,42,68,71-95}$ On the right side of the figure are shown the FFM studies using a colloidal probe made from: collagen; ${ }^{96}$ latex; ${ }^{97}$ polyethylene (PE); ${ }^{25,28,45,98}$ polyethylene glycol (PEG); ${ }^{99}$ poly(methyl methacrylate) PMMA; ${ }^{26,27,95,100}$ polystyrene (PS); ${ }^{33,71,101}$ cellulose; $;^{31,102-109}$ and silica. ${ }^{35,36,98,110-117}$ The volume of the symbol corresponds to the number of studies using the specific system of materials. Colloidal probes of the same material are in groups, while the green-shaded area represents the knowledge gap on friction between soft surfaces that requires future research attention. 

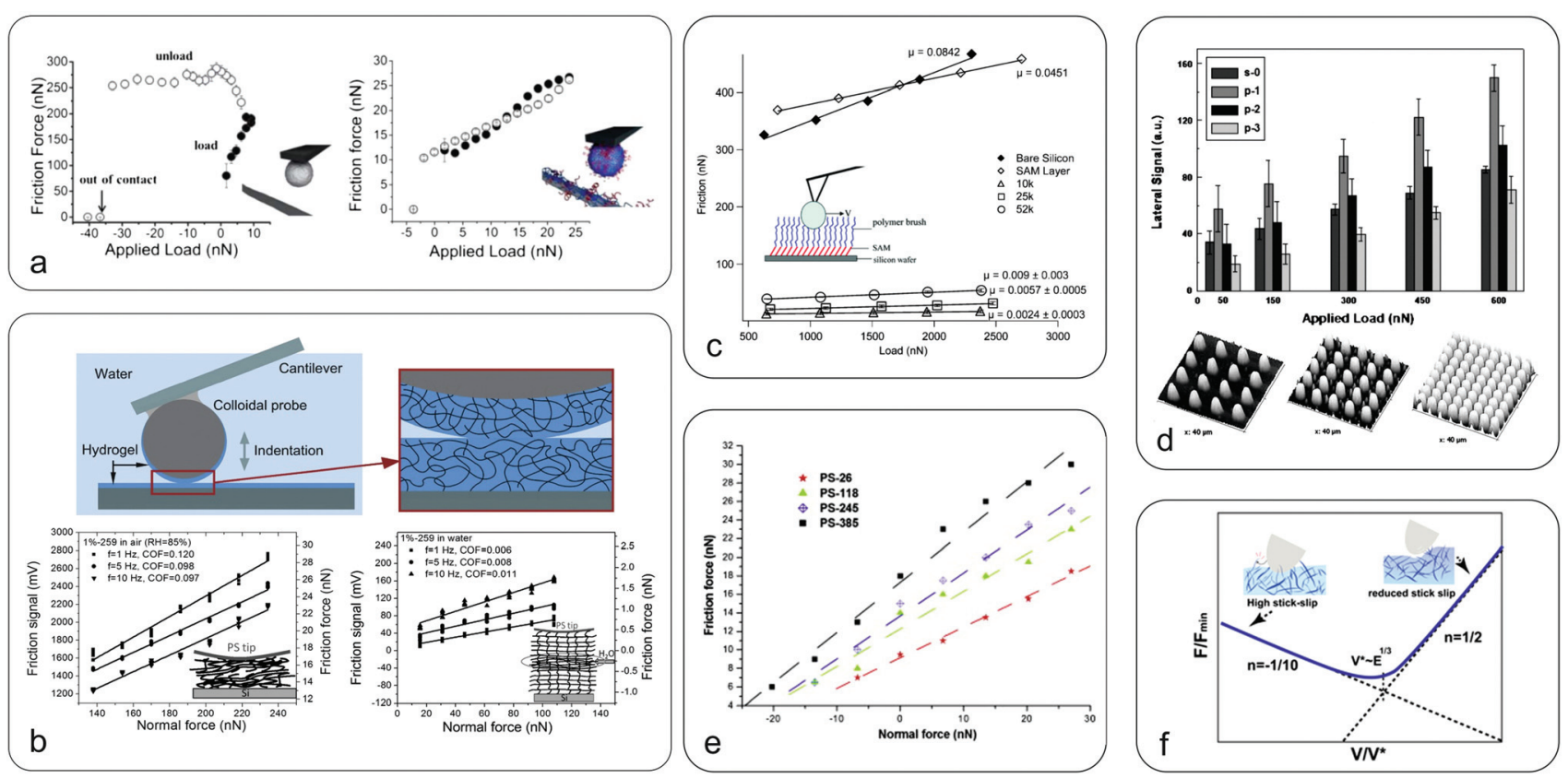

Fig. 5 Nanoscale friction in soft surfaces. (a) Friction as a function of applied load between a bare cellulose probe (left) and nanofibrillated surfaces with grafted CMC-g-PEG polymer brushes (right), highlighting the efficacy of polymer brushes in reducing friction due to electrosteric repulsion, with a 30 times reduction at zero load (republished with the permission of Royal Society of Chemistry, from "Direct measurements of non-ionic attraction and nanoscaled lubrication in biomimetic composites from nanofibrillated cellulose and modified carboxymethylated cellulose", A. Olszewska, J. J. Valle-Delgado, M. Nikinmaa, J. Laine and M. Osterberg, Nanoscale, 2013, 5, 11837-11844, Copyright 2013; ${ }^{104}$ permission conveyed through the Copyright Clearance Center). (b) Friction between a hydrogel-coated (PDMAA-co-MABP) probe and hydrogel surfaces in air (left) and in water (right) at various sliding speeds with the inset showing a schematic representation of the soft contact mechanics (adapted with permission from K. Li, C. K. Pandiyarajan, O. Prucker and J. Ruhe, Macromol. Chem. Phys., 2016, 217, 526-536. ${ }^{33}$ Copyright 2016 John Wiley \& Sons, Inc.). Hydrogel friction is reduced by an order of magnitude once hydrated (hydration lubrication). (c) Friction force versus load for PDMS polymer brushes of different chain lengths with the inset presenting a schematic of the polyethylene bead sliding over polymer brushes (adapted with permission from L. J. Landherr, C. Cohen, P. Agarwal and L. A. Archer, Langmuir, 2011, 27, 9387-9395. ${ }^{118}$ Copyright 2011 American Chemistry Society). The friction is reduced by an order of magnitude for polymer brush coated surfaces as compared to bare silicon or SAM surfaces. However, lower molecular mass chains have reduced friction compared to longer chains, and increasing grafting density reduces friction by creating a more uniform surface. (d) Three-dimensional AFM images of surfaces patterned with pillars of increasing density (bottom) and the corresponding frictional forces against a borosilicate colloidal probe (top) (adapted with permission of Taylor \& Francis Ltd from "Adhesion and friction behavior of positively or negatively patterned polymer surfaces measured by AFM", X. L. Zhang, F. Liu, W. Z. Wang, G. W. Yi and J. H. Jia, J. Adhes. Sci. Technol., 2013, 27, 2603-2614. ${ }^{113}$ ). It shows that increased spacing between the pillars leads to increased friction due to the collision effect between the colloidal probe and the pillars. The $s-0$ refers to a smooth surface, while $p-1, p-2$, and p-3 indicate surface with pillars of increasing density. (e) Friction force for a silicon nitride probe sliding on soft polystyrene surfaces of different molecular weights (reprinted from "Evaluation of nanotribological behavior of amorphous polystyrene: the macromolecular weight effect", A. Ghorbal and A. Ben Brahim, Polym. Test., 2013, 32, 1174-1180. ${ }^{91}$ Copyright 2013, with permission from Elsevier). The friction is increased with increasing molecular weight due to increased interaction and dissipation in the longer polymer chains, similar to panel c. (f) Speed-dependence of friction on polyacrylamide (PAAm) hydrogels (reprinted with permission from T. Shoaib, J. Heintz, J. A. Lopez-Berganza, R. Muro-Barrios, S. A. Egner and R. M. Espinosa-Marzal, Langmuir, 2018, 34, 756-765. ${ }^{112}$ Copyright 2018 American Chemical Society). While initially friction is decreased with increasing velocity due to reducing slip-stick (polymer chain adsorption and release is a kinetic process), when it reaches a transition velocity the friction increases with speed, possibly due to increased deformation of the hydrogel.

materials. It has been found that on polydimethylsiloxane (PDMS) surfaces versus a PE colloidal probe, the coefficient of friction $(\mu)$ is very low when the adhesion is negligible. ${ }^{98}$ However, $\mu$ increases with increasing concentration of pendant PDMS chains that results in larger adhesion and a consequently increased contact area. In another instance, the photodegradation of poly(ethylene terephthalate) (PET) films upon exposure to ultraviolet (UV) light renders the films more hydrophilic, resulting in increased non-covalent attractive forces with a $\mathrm{Si}_{3} \mathrm{~N}_{4}$ probe and, consequently, increases the friction. ${ }^{77}$ In contrast, repulsive interactions can reduce friction as shown in Fig. 5a where micron-sized cellulose spheres slide against a nanofibrillated cellulose surface adsorbed with polyethylene glycol grafted carboxymethyl cellulose (CMC-g-PEG). The aqueous lubrication was mainly attributed to the electrosteric repulsion arising from the high anionic charge by deprotonation of carboxyl groups on CMC overcoming the van der Waals and hydrogen bonding-associated adhesive forces. ${ }^{104,105}$

A key surface interaction that is particularly relevant for soft and biological interfaces in hydration lubrication concerns the effect of water structure up to a few nanometres away from the surface. The behaviour of this layer includes the influence of hydration shells from the surface or surrounding ions, the availability of bulk water and steric disruption by polymer 
chains. It was shown that friction of a polystyrene colloidal probe coated with a PDMAA-co-MABP hydrogel sliding on dry PDMAA-co-MABP hydrogels is high and is reduced with increasing sliding velocity (Fig. 5b). ${ }^{33}$ However, on hydrated hydrogels, friction is significantly lower than that in the dry hydrogels and progressively increases with the sliding velocity due to increasing polymer concentration in the contact area during shearing. This suggests that in hydrated hydrogels the viscous drag of water within the hydrogel at the interface is the main cause of hydration lubrication. Furthermore, at high loads $(>60 \mathrm{nN})$, friction is increased due to the accumulation of the polymer in the contact area arising from hydrogel compression. It has also been shown that ionic functional groups or biopolymeric molecules, such as mucins tethered at the surface can entrap water that cannot be squeezed out but remain labile resulting in better lubrication and reduced adhesion. ${ }^{32,119}$ For a comprehensive understanding of hydration lubrication, readers may refer to thorough reviews by Klein's group. ${ }^{120,121}$

Under ambient non-hydrated conditions, capillary forces arising from a meniscus of water between the probe and surface are another factor that tends to dominate friction. It can significantly increase adhesion and, consequently, friction is strongly dependent on humidity. ${ }^{99}$ It was shown that on hydrophilic silica surfaces, and at low sliding speed, capillary forces provide a major contribution to friction. ${ }^{84}$ However, as the speed increases, $\mu$ is reduced because there is less time for a stable meniscus to build up at the interface. A similar study on mica and silica surfaces against a cellulose probe also showed that capillary adhesion dominates friction but further revealed a hysteresis between loading and unloading frictionload curves, suggesting a larger condensate due to slow evaporation of the formed meniscus. ${ }^{109}$

Individual components in lubricants, such as polymers or proteins, can significantly alter the frictional properties, depending on their ability to adsorb on the surface. For instance, poly(oxyethylene)-poly(oxypropylene)-poly(oxyethylene) (PEO-PPO-PEO) is an effective lubricant on polypropylene (PP) and polyethylene (PE) surfaces but not on cellulose surfaces, which is attributed to the ability of the lubricant to attach to PP and PE but not to cellulose. ${ }^{61}$ Similar results were reported for mucin, which reduces the friction between a PMMA colloidal probe sliding on a PMMA surface. ${ }^{100}$ In contrast, when the lubricating properties of mucin were measured with a sharp $\mathrm{Si}_{3} \mathrm{~N}_{4}$ tip against a PDMS surface, friction was increased as a result of the tip ploughing through the mucin layers adsorbed on the surface. ${ }^{86}$ However, when the tip was rendered hydrophobic with octadecyltrichlorosilane (OTS) the presence of mucin reduced the friction as compared to the bare hydrophobic PDMS because it blocked the strong hydrophobic attractive force. This suggests that surface chemistry at the interface due to adsorption of mucin to the surface can be an important factor that cannot be ignored.

Further evidence that surface chemistry dictates the ability of lubricants to reduce friction was shown upon adsorption of proteoglycans on polycarbonate urethanes (PCU) used in medical devices, and hydrophobic or hydrophilic self- assembled monolayers (SAMs) as a model system. ${ }^{115}$ It was shown that on hydrophilic SAM surfaces, proteoglycan adsorption has a negligible impact on friction, while proteoglycan adsorption on hydrophobic PCU surfaces significantly decreases friction when compared to the bare hydrophobic surfaces. The decrease in friction is even larger on surfacemodified PCU surfaces that are locally softer than PCU surfaces. In contrast, when proteoglycan is adsorbed on hydrophobic SAMs the impact on friction is negligible. Although both SAMs, PCU, and modified PCU are hydrophobic, the difference arises from the higher contact pressure on the nondeformable SAM on gold, compared to lower contact pressure on softer PCU due to the increase in the probe-surface contact area highlighting the impact of surface deformability on the lubricating ability of molecules.

Another interesting strategy adopted by nanotribologists is to graft polymer brushes onto surfaces and use FFM to study the frictional properties. The excellent lubricating properties of polymer brushes arise from the osmotic pressure within the polymer brushes that resist compression, and from the opposition to the entropy loss that would result between two opposing brush-covered surfaces if they were compressed and, therefore, ordered to some degree. ${ }^{21}$ A study on poly(L-lysine)-graftpoly(ethylene glycol) (PLL- $g$-PEG)-modified $\mathrm{SiO}_{2}$ surfaces showed that the solvent environment plays a key role in the frictional properties of polymer brushes; the higher the solvation of the polymer brushes the lower the friction. ${ }^{122}$ This was also shown during the adsorption of chitosan brushes on cellulose surfaces that reduces friction due to electrosteric repulsion arising from the extended chitosan chains. ${ }^{103}$ Friction is reduced as the grafting density of the polymer brushes is increased, which is attributed to the reduced contact with the underlying surface and to interaction with a denser layer with less entanglement in long chains (Fig. 5c). ${ }^{123}$ Furthermore, the longer the length of the grafted polymers chains at a given grafting density the higher the friction, as a result of reduced chain mobility and higher viscosity of the brushes. ${ }^{118}$

\subsection{Effect of surface roughness on nanoscale friction}

As discussed in previous sections, multi-asperity contacts can be treated as single asperity if the contact pressure is sufficient to squeeze out all the asperities at the contact. On softer surfaces, although this requires less force as the Young's modulus is reduced and it is not as prominent as on hard surfaces, surface roughness remains a major factor that affects adhesion and friction. Studies on nano-patterned PDMS and polyimide surfaces have shown reduced adhesion and friction as compared to flat surfaces, as a result of the reduced contact area at the interface (Fig. 5d). ${ }^{113,117}$ Similar results were found in a study that used silica particles attached to a silica surface to control roughness in order to investigate the correlation between surface roughness and frictional properties. ${ }^{28}$ When the particle density on the surface was decreased from 450 particles per $\mu \mathrm{m}^{2}$ to 245 particles per $\mu \mathrm{m}^{2}$ both adhesion and friction were reduced. 
The degree that surface topography will affect the frictional properties is related to the selected scan size during a FFM measurement. For instance, if the scan size is much smaller than surface asperities, then surface topography will have a small impact on frictional properties. Consequently, scan size plays a significant role in studying frictional properties. More specifically, it has been found on cellulose surfaces that for scan sizes smaller than surface asperities, $\mu$ initially decreases with increasing load and eventually becomes load independent. In contrast, for scan sizes larger than surface asperities, $\mu$ is increased with increasing load due to the asperity dominated friction. ${ }^{108}$ Similar studies attributed the scale dependence (microscale to nanoscale) of $\mu$ to surface roughness, and emphasized that friction is scale-dependent and no assumption can hold from one scale to another. ${ }^{85}$

\subsection{Effect of intrinsic material properties on nanoscale friction}

Apart from surface chemistry and topography, friction is greatly influenced by the intrinsic properties of a material, such as its viscoelastic properties. This becomes particularly relevant for soft materials where viscoelastic mechanical loss dominates friction on polymer films. ${ }^{62}$ At the same time, the interaction between a surface and a probe can alter the properties of the material. Several groups have used FFM on soft surfaces to study the glass transition temperature $\left(T_{\mathrm{g}}\right)$, the surface relaxation, and the molecular motion of polymeric films. Above the $T_{\mathrm{g}}$ a polymer in a rigid state changes to a rubbery state. It has been found that $T_{\mathrm{g}}$ is much lower on the surface of PS films than the bulk value, which could be an indication of a greater free volume at the surface of the polymer. ${ }^{62,64}$ The reduction in $T_{\mathrm{g}}$ is even larger at lower molecular weights, which can be explained by an excess free volume at the surface..$^{63}$ During FFM experiments, the applied load and scanning velocity can also affect the $T_{\mathrm{g}}$. It has been found on PS that when the applied load is small enough and not sufficient to disturb the surface then the $T_{\mathrm{g}}$ is shifted to higher temperature with increasing speed. ${ }^{92}$ However, at high loads the $T_{\mathrm{g}}$ is shifted to lower temperatures with increasing speed due to the heating effect, where the energy dissipated through friction increases the temperature locally and increases the molecular motion. Since friction in polymers is affected by the $T_{\mathrm{g}}$, polymer blends can reveal different frictional properties, so the effect is entirely dependent on the detailed response of the polymers under study. For instance, for poly(vinyl methylether) (PVME) and PP blends, an increase in $T_{\mathrm{g}}$ increases the friction, which is explained by a greater loss in dissipation energy. ${ }^{73}$

Similar to $T_{\mathrm{g}}$, the activation energies for $\alpha$ - and $\beta$-relaxations, which are related to the translation of the molecule through the medium and the change in molecular conformation respectively, can be different at a polymer surface as compared to its bulk value. More specifically, it has been found that the apparent activation energies for $\alpha$ - and $\beta$-relaxations are smaller at the surface of PS than its bulk value, which indicates a significant increase in the molecular motion at the surface. ${ }^{62,64,92}$ Similar results were found on
PMMA films, where the activation energies for $\alpha$ - and $\beta$-relaxation were found to be three times lower on its surface compared to the bulk values. ${ }^{68}$ These results reveal a greater free volume and a higher molecular mobility at the polymer/ air interface. The observed reduction in surface relaxation can be enhanced by the high stress from the sharp tips that activate $\alpha$ - and $\beta$-transition at temperatures below the glass transition temperature. ${ }^{35}$ Since decreased molecular mobility results in reduced friction, it is expected that ultrathin polymer films may have different frictional properties from thicker films. This was shown with ultrathin PVME films, where the friction is reduced with decreasing film thickness as a consequence of the reduced polymer chain mobility and the increasing polymer stiffness arising from the confinement of the polymer chains. ${ }^{73}$

The molecular weight (MW) of a polymer can have a significant impact on the interactions between the sliding body and, thus, its frictional properties. More specifically, it has been found that the friction on PS surfaces increases with the length of PS polymer chains, which is attributed to larger adhesion forces as a result of increasing interactions with polymer chains, mainly through van der Waals and acid-base interactions (Fig. 5e) ${ }^{91}$ Apart from the MW, the degree of crosslinking can also affect friction. Sliding of colloidal silica particles on polyacrylamide (PAAm) hydrogels, with Young's moduli of $2 \mathrm{kPa}, 9 \mathrm{kPa}$, and $40 \mathrm{kPa}$, reveals two different boundary lubrication mechanisms (Fig. 5f). ${ }^{112}$ Initially, the friction decreases with increasing speed until it reaches a transition velocity where the friction starts to increase with increasing speed. Below the transition speed, the decrease in friction with increasing sliding speed is related to the continuous adsorption and desorption of the polymer chains onto the sliding body; the polymer chains that were adsorbed on the counter surface require more time to re-adsorb once the contact breaks due to the sliding motion. It was found that less cross-linked PAAm hydrogels exhibit reduced friction due to the larger relaxation time of the polymer chains, requiring more time for re-adsorption. At the same time, although the mechanism for the regime above the transition speed where friction increases with speed is not elucidated, it was found that less cross-linked PAAm hydrogels favour the transition into that regime. It can be seen that as the degree of crosslinking is reduced, friction is also reduced, at least for low sliding speeds. This was also shown in ultrathin PDMS films where $\mu$ was lower than in thicker films, which is a consequence of poor cross-linking in ultrathin films. ${ }^{25}$

Chain entanglement was found to have a similar effect on crosslinking in reducing friction but due to a different mechanism. More specifically, it was found that on PS and PMMA surfaces, friction is increased with increasing MW up to a value close to the critical MW for entanglement in bulk PS and PMMA, after which the effect of MW on $\mu$ is small. ${ }^{83}$ It was suggested that at low and up to a critical MW, the AFM tip is ploughing between polymer chains during FFM. On the onset of entanglement, the energy dissipation mechanism changes and the tip is sticking in the loops between chains and is 
pulling them until they break. Reduction of friction was also observed when PMMA was modified upon exposure to UV light, which was correlated to a gradual reduction in MW and, thus, chain entanglement. ${ }^{83}$

\subsection{Effects of experimental conditions on nanoscale friction}

Sliding speeds and loads used during FFM are critical factors in the measured $\mu$. For instance, at high velocities, friction on silica is dominated by contacting asperities. ${ }^{84}$ Similarly, in PMMA beyond a critical velocity, friction is increased due to the deformation of its surface and higher energy dissipation. ${ }^{84}$ However, soft surfaces such as PDMS can absorb the asperity impact without causing plastic deformation, resulting in constant friction even at high velocities. ${ }^{84}$ For soft surfaces, the load has a major impact on friction. Studies with a cellulose probe against silica surfaces showed that at low loads (60-80 $\mathrm{nN}$ ), the friction-load relationship is linear, indicating that the contact cannot be treated as a single asperity. ${ }^{31}$ In contrast, at higher loads the cellulose-based colloidal probe is deformed and can be treated as a single asperity, while the dependency of friction on normal load follows a sublinear relationship. Load and, consequently, plastic deformation can affect friction both at the macro- and nanoscale as was shown in a study on PS surfaces where friction yielded similar values at both scales, suggesting a similar macro and nanotribological mechanism. ${ }^{93}$

In another study, focusing on poly( $N$-isopropylacrylamide $)$ brushes, Ramakrishna et al. ${ }^{124}$ studied the effect of scanning distance on the measured friction forces. They showed that when the polymer chains are highly swollen and the sliding distance is smaller compared to the swollen brush thickness, the measured friction is mainly due to the backand-forth lateral bending and stretching of the chains and the kinetic friction is only measured when the sliding distance overcomes the bending and stretching of the chains. However, in the case of collapsed polymer chains, no effect of sliding distance was observed on the measured friction. Other external stimuli, such as temperature and $\mathrm{pH}$, can also have a significant impact on the frictional properties of a system and readers can refer elsewhere for further insight. ${ }^{125-127}$

\section{Molecular dynamics simulations complementing FFM}

Friction force microscopy has made a significant breakthrough in exploring frictional phenomena occurring at the nanoscale. Still, achieving a detailed understanding on phenomena and mechanisms such as those occurring at the tip-substrate interface has not yet been fully achieved. Molecular dynamics (MD) simulations, which use Newton's law and empirical potentials to calculate the interactions between atoms and predict their trajectories and behaviour, have often proved to be a highly suitable complementary technique to shed light on friction at atomic and molecular levels. Although there has been a comprehensive review focussing on how MD simulation can elucidate the mechanisms of atomic friction during hard-hard interactions, ${ }^{128}$ which are briefly discussed here, we focus on interactions between hard-on-soft and soft-on-soft surfaces.

MD studies have been used to establish friction laws in dry nanoscale contacts. ${ }^{50}$ It has been found that nanoscale friction is highly sensitive to contact mechanics, but single-asperity theories break down at the nanoscale, and friction ultimately depends linearly on the number of atoms that are interacting at the interface. While the frictional force versus load relation is non-linear for adhesive surfaces, a transition to a linear relation takes place as adhesion is decreased, which is consistent with the experimental results at the nanoscale as discussed above in section 3 . The transition takes place when the contact roughness becomes large as compared to the interactions at the interface, such as when the sliding interface is damaged and becomes rougher. MD studies have been mainly used to shed light on atomic friction phenomena at hard-hard contacts. For instance, MD was used in parallel with FFM to observe friction between a platinum AFM tip sliding on $\mathrm{Au}$ (111). ${ }^{129}$ Both FFM and MD revealed stick-slip behaviour, proving $\mathrm{MD}$ as a reliable approach to interpret AFM data, while it further revealed that atomic stick-slip is thermally activated at low speeds. Similar MD studies ${ }^{128,130}$ on how the substrate crystal lattice affects friction revealed that stick-slip and superlubricity could be a result of lattice mismatch, as was elegantly presented experimentally by Dienwiebel et $a .^{29}$ (Fig. 1). This role of lattice incommensurability then leads to further insights, impossible to achieve with current FFM, on the role of surface shear stress distributions at the interface ${ }^{131}$ in these matched or mismatched lattices, and its influence on the stick-slip mechanism.

Another aspect of friction that MD simulations have complemented FFM is hydration friction, as shown in an investigation into friction on graphite, both under vacuum and in water. ${ }^{132}$ While both FFM and MD showed that water has a negligible impact on friction for loads larger than $5 \mathrm{nN}$, MD simulations revealed the role of the hydration layer at the graphene/water interface, which could not be achieved by FFM alone. Similar experiments showed that although the presence of water does not affect friction at flat surfaces, at atomic step edges (such as stepped graphite surfaces) the friction is significantly increased. ${ }^{133}$ Another MD study, using two bilayers of decanol molecules separated by water, investigated the role of water and hydration friction in nanoconfinement. ${ }^{134}$ It was found that three friction regimes are present with decreasing water thickness; (1) for thick water films, friction is governed by bulk water viscosity, (2) for water films of about $1 \mathrm{~nm}$, the interfacial layer is highly viscous and increases friction, and (3) at the dry friction limit, the interfacial slip sets in.

As discussed above the roughness of a surface can significantly affect friction, since it affects the contact area between the tip and substrate, and MD simulations have been used to study this area. A series of MD simulations was used to study the frictional behaviour of nanopatterned silicon surfaces and how nanopatterning can be used to tune friction at the nanoscale. ${ }^{135}$ It was found that for nanopatterned surfaces, there is always a linear dependence of load on the frictional forces, 
both for adhesive and non-adhesive surfaces, which is independent of the nanopattern geometry. This can represent Amontons' law (eqn (1)) and it's relation to the real contact area. In contrast, flat surfaces exhibit a non-linear relationship between frictional force and load when adhesion is introduced in the system. It was also found that friction can be tuned by adjusting the nanopattern period and, thus, nanopatterning can be used to control adhesion and friction at the nanoscale. Another MD study examined the friction characteristics at the nanoscale between multi-asperity tips and textured surfaces. ${ }^{136}$ It was found that the number of asperities on the tip and the contact area with the substrate can significantly affect friction and cause various degrees of damage to the surface, while the ratio between the size of asperities and surface texture width is an important parameter that influences friction.

FFM can probe the friction force between a tip and a substrate, but it is difficult to recognise whether the deformation at the interface is plastic or elastic, or even whether ploughing is occurring, and this is an area in which MD can provide significant wealth of information. For instance, MD simulations allowed the study of a nanometric scratching process, where a rigid diamond sphere is sliding on face-centred cubic (fcc) single crystal copper. This enabled the first determination of the ploughing friction coefficient and the adhesion friction coefficient at the nanoscale. ${ }^{137}$ It was found that macroscale theory slightly overestimated the ploughing friction coefficient at the nanoscale, while the adhesion friction coefficient was independent of the indentation depth and was almost stable.

Although MD simulations have mostly been used for hardhard contact surfaces, the gradual shift to hard-on-soft is demonstrated in one of the most recent MD studies, where a rigid indenter of $5 \mathrm{~nm}$ radius was simulated sliding over an amorphous polyethylene. ${ }^{43}$ It was found that friction is mainly composed of a plough force (cohesive zone) and an adhesion force (interface zone). Elastic deformation was attributed to van der Waals interactions in the cohesive zone, while bond angle energy and dihedral energy of the molecular chain dominated plastic deformation. Also, the presence of attractive interactions significantly increased the friction, as compared to repulsive interactions between the indenter and soft polyethylene, while the higher the indentation the larger the contribution of the plough force and hence the larger the friction.

Besides soft surfaces, MD simulations have been effectively used to interpret the FFM results on soft polymer-coated surfaces. A MD study on polymer-polymer interface friction revealed three different mechanisms governing frictional behaviour and deformation; ${ }^{138}$ interfacial "brushing", which has the major contribution, followed by "combing" and "chain scission". These mechanisms refer to how the polymer chains are interacting with each other, either with a small section ("brushing"), sliding between chains ("brushing") or even breakage of the chains when their path is distracted ("chain scission"). The same study also revealed three regimes, ranging from periodic stick-slip at low sliding speeds to irregular stick-slip and dynamic frictional sliding as the sliding speed increases. Immiscible polymer brush systems can greatly reduce dissipation, as was shown on PMMA (immersed in acetophenone) and PNIPAM (immersed in water) brushed surfaces in a combination FFM and MD study. ${ }^{139}$ It was reported that friction between PMMA-PNIPAM surfaces (immiscible system) was significantly lower than that between PMMA-PMMA (miscible system), while they have similar loadbearing capacity. FFM and MD were also used to study the effect of crosslinking on the tribological behaviour of polymer brushes. ${ }^{140}$ It was found that not only does $\mu$ increase with the degree of crosslinking, but also that the length of the crosslinker can affect $\mu$ with higher length leading to a decreased friction. In summary, complementing FFM with MD simulations has already started to advance our understanding of friction in soft surfaces and we expect that combining FFM experiments and MD simulations will become a standardized approach to address biophysical questions and improve interpretation of nanofrictional mechanisms in the future.

\section{Applications}

The relentless increase in the number of recent studies using FFM to quantify frictional forces has largely been fuelled by the enormous practical and technological questions in biological systems such as joints, cells, contact lenses, saliva-coated oral mucosa and cosmetic applications. In this section, we cover a few important examples of recent biomedical, biological and technological applications of friction measurements at the nanoscale that have largely used hard-on-soft and soft-onsoft contact surfaces.

\subsection{Cartilage}

Articular cartilage has a very low coefficient of friction and is essential for joint motion, which when damaged can lead to osteoarthritis. The understanding of friction and wear behaviour of cartilage, as well as factors that affect it such as age, is valuable in treating cartilage-related problems. FFM has been successfully used to quantify friction in boundary conditions with appropriate test conditions that allow the negation of hydrostatic pressurization, which supports approximately $90 \%$ of the load in joints. ${ }^{36}$ Upon measuring friction on murine cartilage using a borosilicate colloidal probe it was revealed that four possible mechanisms contribute to boundary friction; interfacial friction that arises from molecular interactions between surfaces, internal friction attributed to inelastic deformation and recovery of material, ploughing friction arising when a hard asperity is sliding against a soft material and causes asymmetric pressure distribution by pushing the material forward, and friction due to collision of the probe with asperities on a rough surface. The study reports that the major component of friction was interfacial shear, while the other three mechanism had only a small contribution.

Although articular cartilage on its own has a low boundary friction coefficient, FFM has clearly uncovered that synovial fluid and its components are the major lubricants. For instance, articular cartilage coated with protein components 
exhibited low friction, whereas when the surface was treated with proteolytic enzymes such as trypsin hydrolysing the protein film, the friction increased. ${ }^{141}$ It is noteworthy that load-bearing regions of articular cartilage exhibit lower friction than non-load-bearing regions, indicating the presence of boundary lubricants that protect from wear and tissue degeneration when joints are starved from fluid lubrication. ${ }^{56}$ The distinct role of synovial fluid components in lubrication has been elegantly studied using hydrophobic and hydrophilic surfaces. ${ }^{142}$ It was reported that lubricin lubricates the hydrophobic surfaces effectively, while it slightly increases friction when inserted between hydrophilic surfaces. The impact of hyaluronic acid (HA) on lubrication was found to be considerably smaller, while no synergistic effect was found between lubricin and HA in terms of lubrication. FFM has also been used to study the increase in friction with the progression of osteoarthritis. It was found that $\mu$ of human femoral head cartilage increased substantially from 0.119 at Stage 0 to 0.409 at Stage 3, and this was also correlated with an increase in roughness, ${ }^{44}$ suggesting a decrease in the presence of friction-reducing protein.

\subsection{Cells}

Since friction in cells is encountered in a plethora of biophysical processes, such as blood flow, cartilage lubrication, cell adhesion and migration, understanding the frictional properties of cells is of great interest. For instance, FFM employing a borosilicate colloidal probe was used to study the frictional behaviour of individual vascular smooth cells. ${ }^{110}$ It revealed that $\mu$ was increased with increasing cellular crosslinking and decreased by cytoskeletal polymerization, which could be used to improve the design in applications of intravenous devices such as stents and heart valves. Furthermore, by directly measuring friction on cells, one could better understand the disorder related to stresses in cells, such as diagnosis of heart diseases. For instance, FFM using a sharp silicon tip was utilised to observe the lateral contraction forces of living cardiomyocytes. ${ }^{3}$ It was found that FFM was able to accurately detect the contraction of these cells, as well as the effect that a drug such as ibutilide has on those cells. Consequently, FFM could be used as a screening test for drugs, in order to understand their mechanics and promote the design of improved drugs.

\subsection{Proteins}

FFM has been widely used to understand molecular interactions between biological molecules, such as protein-carbohydrate interactions, which are important in cellular recognition processes. For instance, by attaching a lectin protein to an AFM probe and sliding it on glass surfaces with immobilized carboxypeptidase Y, FFM was used to successfully study the dissociation mechanics and kinetics of the molecular complex. ${ }^{143}$ Similar work was performed on proteins to investigate the interaction between human immunoglobulin (IgG) and anti-human IgG. ${ }^{144}$ The results revealed differences in their interaction when using different preparation methods and confirmed the specific interactions between antigen and antibody, making FFM a valuable tool for the development of drugs or screening tests. Recently, FFM coupled with macroand micro-scale tribology and theoretical analysis was used in our laboratory $^{2}$ to study the synergistic interactions of the salivary proteins that are responsible for the outstanding lubrication properties of the salivary pellicle coating the oral mucosa. An electrostatically-driven self-assembly between negativelycharged mucin and non-mucinous positively charged proteins explained the boundary and viscous lubrication properties of the salivary film. ${ }^{2}$ A fundamental understanding of the mechanism of salivary lubrication offers the potential for rationally designing an optimally performing salivary substitute that could improve the quality of life of people suffering from xerostomia caused due to age, polypharmacy, immune diseases, etc.

\subsection{Contact lenses}

Measuring the friction properties of soft contact lenses has gained significant research momentum over the past few years due to growing concerns with contact lens-associated discomfort, with Kim et al. $^{32}$ revolutionizing the use of FFM for soft hydrogels (Fig. 1). In an interesting FFM study, they used a polystyrene colloidal probe to study friction on poly(2-hydroxyethyl methacrylate) (pHEMA) hydrogel lenses, finding that ionic functional groups at the surface of the lenses can lower the friction, which was attributed to the interactions of water and orientation of hydrogel chains. ${ }^{32}$ The study also showed that when fully hydrated, extended non-crosslinked chains can increase friction, but when partially dehydrated the chains collapse and friction is reduced.

\subsection{Cosmetics}

FFM is finding increasing use in cosmetics to study friction and adhesion on skin and hair, as well as the impact of formulations such as skin creams and hair conditioners. For instance, FFM was used to study the frictional properties of skin, both virgin and damaged, and how it is improved by skin cream-treatment. ${ }^{145,146}$ It was found that skin cream decreases roughness and smoothens the skin both for virgin and damaged skin, while it also increases its hydrophilic properties. As one might expect, $\mu$ was higher on damaged hair, but it was also higher in liquid than in air, which was partially attributed to the deformation of hair due to water absorption. ${ }^{89}$ Using colloidal AFM probes against hair, conditioners have been found to reduce friction both in virgin and damaged hair (although the reduction in friction is much higher in the latter), ${ }^{88}$ while anisotropic frictional properties on the cuticle surfaces of hair that are attributed to the striations present on the hair and parallel to the long-axis of the hair have also been reported. ${ }^{147}$

\subsection{Fabrics}

Understanding friction between fabric fibres, such as cotton, is essential for various industrial and household applications. Reduced friction between fibres can extend their lifetime, while friction between fibres and skin is an important factor 
on how we perceive and evaluate the quality of a product. An FFM study on the frictional properties of cotton fibres and how a fabric conditioner affects it showed that increased roughness results in increased $\mu$, while the presence of fabric conditioner reduces $\mu .{ }^{95}$ Similarly, in the papermaking and book industry, friction between fibres is an important factor in holding together the fibre network, while friction between paper and machine parts is of similar importance in applications such as printing. Friction measurements between pulp fibres revealed that surface roughness greatly affects their frictional properties because the friction coefficient increases. ${ }^{148}$

\section{Conclusions and future perspectives}

As a technique, FFM on soft surfaces presents a new paradigm in nanotribology, demonstrating a growing importance with the incorporation of soft materials into electronics and robotics. At a fundamental level, FFM plays a crucial analytical role in understanding nanoscale interactions at biological interfaces, in addition to the increasing demands of traditional soft material industries (e.g. food, healthcare, biomedical, agrochemical, cosmetics) among many others. Although FFM first emerged in 1987, the work that has been done so far on soft surfaces is fairly restricted, with the focus being on characterizing the intrinsic properties of soft polymeric surfaces, such as relaxation of polymers and glass transition phenomena that play an important role in frictional properties. The introduction of colloidal probes for friction measurements was a major breakthrough for investigating friction on soft surfaces, leading to much progress in understanding surface interactions in materials ranging from hard metallic spheres to softer polymeric probes. Still, most of the published studies on friction using FFM are utilizing conventional polymeric probes with an elastic modulus higher than $1 \mathrm{GPa}$ and there are relatively rare instances where a system of soft probe/soft surface has been used to measure lateral forces.

Designing colloidal probes that exploit the parallel developments in material chemistry can help to address many fundamental research challenges. The investigation of this knowledge gap, regarding the capability of performing nanotribology experiments with elastic moduli ranging from tens of $\mathrm{kPa}$ to few MPa at the nanoscale, will be of great importance, and will find use in a wide range of future biological and technological applications, where soft materials with desired frictional properties are in demand. However, as has been demonstrated, there are many unresolved challenges in this field, with many complex interactions. For instance, the elastic modulus of a material surface can be different from its bulk value which could cause inconsistencies in the measurements, while the direct comparison between macro and nanoscales is complicated by the length scale of surface roughness and asperity contact, and the measurement itself has been shown to change the relaxation state of the soft polymeric surface. Furthermore, due to the low elastic modulus, the conditions of the well-established contact mechanics models may not be met and, consequently, there may be inconsistencies in the interpretation of the measured data across the literature. The utilisation of advanced fabrication methods, such as electronbeam lithography, will be a fascinating avenue to deliver concrete breakthroughs in measuring the interfacial friction of soft sliding nanostructured surfaces with well-defined roughness. Also combining experimental frictional measurements with continuing advances in molecular dynamics studies will be increasingly informative, allowing the rapid determination of the molecular scale mechanisms governing soft tribology. Nanoscale friction in hydrogel-based and biomaterial-based colloidal probes that emulate biological surfaces with relevant modulus and roughness offers excellent opportunities for future interdisciplinary research involving material scientists, biomaterial engineers, mechanobiologists, nanotribologists, and physicists. Such fundamental knowledge is key to eventually design a new generation of soft materials with the desired frictional properties that will tackle a variety of global challenges, from reduction in energy consumption to biological tissue repair.

\section{Conflicts of interest}

There are no conflicts to declare.

\section{Acknowledgements}

Funding from the European Research Council (ERC) under the European Union's Horizon 2020 research and innovation programme (grant agreement no. 757993) is acknowledged.

\section{References}

1 J. Cumings and A. Zettl, Science, 2000, 289, 602-604.

2 F. Xu, E. Liamas, M. Bryant, A. F. Adedeji, E. AndabloReyes, M. Castronovo, R. Ettelaie, T. V. J. Charpentier and A. Sarkar, Adv. Mater. Interfaces, 2019, 1901549.

3 Y. S. Liu, J. T. Feng, L. Shi, R. B. Niu, Q. M. Sun, H. Liu, J. Li, J. H. Guo, J. H. Zhu and D. Han, Nanoscale, 2012, 4, 99-102.

4 A. Sarkar, E. Andablo-Reyes, M. Bryant, D. Dowson and A. Neville, Curr. Opin. Colloid Interface Sci., 2019, 39, 6175.

5 V. Rubino, A. J. Rosakis and N. Lapusta, Nat. Commun., 2017, 8, 15991.

6 Z. Burton and B. Bhushan, Nano Lett., 2005, 5, 1607-1613.

7 V. Bormuth, V. Varga, J. Howard and E. Schaffer, Science, 2009, 325, 870-873.

8 M. Urbakh, J. Klafter, D. Gourdon and J. Israelachvili, Nature, 2004, 430, 525-528.

9 M. Kappl, F. Kaveh and W. J. Barnes, Bioinspiration Biomimetics, 2016, 11, 035003. 
10 A. Socoliuc, E. Gnecco, S. Maier, O. Pfeiffer, A. Baratoff, R. Bennewitz and E. Meyer, Science, 2006, 313, 207-210.

11 B. Bhushan, J. N. Israelachvili and U. Landman, Nature, 1995, 374, 607-616.

12 O. Hod, E. Meyer, Q. Zheng and M. Urbakh, Nature, 2018, 563, 485-492.

13 M. Krieg, G. Fläschner, D. Alsteens, B. M. Gaub, W. H. Roos, G. J. L. Wuite, H. E. Gaub, C. Gerber, Y. F. Dufrêne and D. J. Müller, Nat. Rev. Phys., 2018, 1, 41-57.

14 R. Bennewitz, Mater. Today, 2005, 8, 42-48.

15 Y. F. Dufrene, T. Ando, R. Garcia, D. Alsteens, D. MartinezMartin, A. Engel, C. Gerber and D. J. Muller, Nat. Nanotechnol., 2017, 12, 295-307.

16 C. M. Mate, G. M. McClelland, R. Erlandsson and S. Chiang, Phys. Rev. Lett., 1987, 59, 1942-1945.

17 G. Toikka, R. A. Hayes and J. Ralston, J. Adhes. Sci. Technol., 1997, 11, 1479-1489.

18 N. Manini, G. Mistura, G. Paolicelli, E. Tosatti and A. Vanossi, Adv. Phys.: X, 2017, 2, 569-590.

19 J. P. Gong, Soft Matter, 2006, 2, 544-552.

20 P. Mocny and H. A. Klok, Mol. Syst. Des. Eng., 2016, 1, 141-154.

21 E. M. Benetti and N. D. Spencer, Helv. Chim. Acta, 2019, 102, e1900071.

22 G. Binnig, C. F. Quate and C. Gerber, Phys. Rev. Lett., 1986, 56, 930-933.

23 R. M. Overney, H. Takano, M. Fujihira, W. Paulus and H. Ringsdorf, Phys. Rev. Lett., 1994, 72, 3546-3549.

24 M. Hirano, K. Shinjo, R. Kaneko and Y. Murata, Phys. Rev. Lett., 1991, 67, 2642-2645.

25 L. J. T. Landherr, Q. Zhang, C. Cohen and L. A. Archer, J. Polym. Sci., Part B: Polym. Phys., 2008, 46, 1773-1787.

26 A. Raj, M. Wang, C. Liu, L. Ali, N. G. Karlsson, P. M. Claesson and A. Dedinaite, J. Colloid Interface Sci., 2017, 495, 200-206.

27 J. An, C. Jin, A. Dedinaite, J. Holgersson, N. G. Karlsson and P. M. Claesson, Langmuir, 2017, 33, 4386-4395.

28 S. N. Ramakrishna, P. C. Nalam, L. Y. Clasohm and N. D. Spencer, Langmuir, 2013, 29, 175-182.

29 M. Dienwiebel, G. S. Verhoeven, N. Pradeep, J. W. Frenken, J. A. Heimberg and H. W. Zandbergen, Phys. Rev. Lett., 2004, 92, 126101.

30 T. R. Matzelle, C. Herkt-Bruns, L. A. Heinrich and N. Kruse, Surf. Sci., 2000, 454, 1010-1015.

31 G. Bogdanovic, F. Tiberg and M. W. Rutland, Langmuir, 2001, 17, 5911-5916.

32 S. H. Kim, A. Opdahl, C. Marmo and G. A. Somorjai, Biomaterials, 2002, 23, 1657-1666.

33 K. Li, C. K. Pandiyarajan, O. Prucker and J. Ruhe, Macromol. Chem. Phys., 2016, 217, 526-536.

34 W. A. Ducker, T. J. Senden and R. M. Pashley, Nature, 1991, 353, 239-241.

35 J. Sondhauss, M. Lantz, B. Gotsmann and A. Schirmeisen, Langmuir, 2015, 31, 5398-5405.

36 J. M. Coles, J. J. Blum, G. D. Jay, E. M. Darling, F. Guilak and S. Zauscher, J. Biomech., 2008, 41, 541-548.
37 I. M. Hutchings, Wear, 2016, 360, 51-66.

38 H. Holscher, A. Schirmeisen and U. D. Schwarz, Philos. Trans. R. Soc., A, 2008, 366, 1383-1404.

39 F. P. Bowden and L. Young, Research, 1950, 3, 235-237.

40 F. P. Bowden and D. Tabor, The friction and lubrication of solids, Clarendon Press, Oxford, Oxford, 1950.

41 J. Y. Park and M. Salmeron, Chem. Rev., 2014, 114, 677-711.

42 C. R. Hurley and G. J. Leggett, Langmuir, 2006, 22, 41794183.

43 S. Zhan, H. Xu, H. Duan, L. Pan, D. Jia, J. Tu, L. Liu and J. Li, Soft Matter, 2019, 15, 8827-8839.

44 S. S. Lee, C. T. Duong, S. H. Park, Y. Cho, S. Park and S. Park, Proc. Inst. Mech. Eng., Part H, 2013, 227, 129-137.

45 M. Roba, M. Naka, E. Gautier, N. D. Spencer and R. Crockett, Biomaterials, 2009, 30, 2072-2078.

46 R. W. Carpick and M. Salmeron, Chem. Rev., 1997, 97, 1163-1194.

47 K. Busuttil, M. Geoghegan, C. A. Hunter and G. J. Leggett, J. Am. Chem. Soc., 2011, 133, 8625-8632.

48 K. L. Johnson, K. Kendall and A. D. Roberts, Proc. R. Soc. London, Ser. A, 1971, 324, 301-313.

49 B. V. Derjaguin, V. M. Muller and Y. P. Toporov, J. Colloid Interface Sci., 1975, 53, 314-326.

50 Y. Mo, K. T. Turner and I. Szlufarska, Nature, 2009, 457, 1116-1119.

51 J. Drelich, Miner. Metall. Process., 2006, 23, 226-232.

$52 \mathrm{~J} . \mathrm{N}$. Israelachvili, Intermolecular and surface forces, Academic Press, Burlington, MA, 3rd edn, 2011.

53 J. Y. Park and P. A. Thiel, J. Phys.: Condens. Matter, 2008, 20, 314012.

54 D. Maugis, J. Colloid Interface Sci., 1992, 150, 243-269.

55 R. W. Carpick, D. F. Ogletree and M. Salmeron, J. Colloid Interface Sci, 1999, 211, 395-400.

56 S. M. T. Chan, C. P. Neu, K. Komvopoulos and A. H. Reddi, J. Biomech., 2011, 44, 1340-1345.

57 D. Qin, Y. N. Xia and G. M. Whitesides, Nat. Protoc., 2010, 5, 491-502.

58 J. Huang, B. Cusick, J. Pietrasik, L. Wang, T. Kowalewski, Q. Lin and K. Matyjaszewski, Langmuir, 2007, 23, 241-249.

59 S. H. Kim, C. Marmo and G. A. Somorjai, Biomaterials, 2001, 22, 3285-3294.

60 X. K. Gu and G. J. Wang, Appl. Surf. Sci., 2011, 257, 19521959.

61 Y. Li, O. J. Rojas and J. P. Hinestroza, Ind. Eng. Chem. Res., 2012, 51, 2931-2940.

62 J. A. Hammerschmidt, W. L. Gladfelter and G. Haugstad, Macromolecules, 1999, 32, 3360-3367.

63 T. Kajiyama, Macromol. Res., 2007, 15, 109-113.

64 T. Kajiyama, K. Tanaka and A. Takahara, J. Polym. Sci., Part A: Polym. Chem., 2004, 42, 639-647.

65 S. Sills and R. M. Overney, Phys. Rev. Lett., 2003, 91, 095501.

66 F. Dinelli, C. Buenviaje and R. M. Overney, J. Chem. Phys., 2000, 113, 2043-2048.

67 S. E. Morgan, R. Misra and P. Jones, Polymer, 2006, 47, 2865-2873. 
68 E. Tocha, H. Schonherr and J. Vancso, Soft Matter, 2009, 5, 1489-1495.

69 M. A. Lantz, D. Wiesmann and B. Gotsmann, Nat. Nanotechnol., 2009, 4, 586-591.

70 R. W. Carpick, D. Y. Sasaki and A. R. Burns, Tribol. Lett., 1999, 7, 79-85.

71 M. Targosz-Korecka, K. E. Malek-Zietek, G. D. Brzezinka and M. Jaglarz, Scanning, 2016, 38, 654-664.

72 W. Ma, Y. Sun, D. Han, W. Chu, D. Lin and D. Chen, Microsc. Res. Tech., 2006, 69, 784-793.

73 D. Wang and H. Ishida, C. R. Chim., 2006, 9, 90-98.

74 G. Haugstad, W. L. Gladfelter and R. R. Jones, Langmuir, 1998, 14, 3944-3953.

75 G. Haugstad, W. L. Gladfelter, E. B. Weberg, R. T. Weberg, T. D. Weatherill and R. R. Jones, Mater. Sci. Eng., C: Biomimetic Mater., Sens. Syst., 1995, 3, 85-89.

76 J. A. Hammerschmidt, B. Moasser, W. L. Gladfelter, G. Haugstad and R. R. Jones, Macromolecules, 1996, 29, 8996-8998.

77 C. R. Hurley and G. J. Leggett, ACS Appl. Mater. Interfaces, 2009, 1, 1688-1697.

78 B. D. Beake, G. J. Leggett and P. H. Shipway, Surf. Interface Anal., 1999, 27, 1084-1091.

79 W. K. Lee, Polymer, 1999, 40, 5631-5636.

80 W. K. Lee, Polym. Test., 2004, 23, 101-105.

81 Y. Fujii, T. Nagamura and K. Tanaka, J. Phys. Chem. B, 2010, 114, 3457-3460.

82 Y. Fujii, K. I. Akabori, K. Tanaka and T. Nagamura, Polym. J., 2007, 39, 928-934.

83 T. J. Whittle and G. J. Leggett, Langmuir, 2009, 25, 22172224.

84 N. S. Tambe and B. Bhushan, Ultramicroscopy, 2005, 105, 238-247.

85 N. S. Tambe and B. Bhushan, Nanotechnology, 2004, 15, 1561-1570.

86 N. Nikogeorgos, J. B. Madsen and S. Lee, Colloids Surf., B, 2014, 122, 760-766.

87 C. L. Chang, T. H. Ho and T. H. Fang, Sens. Mater., 2017, 29, 1589-1597.

88 C. LaTorre and B. Bhushan, J. Vac. Sci. Technol., A, 2005, 23, 1034-1045.

89 N. Nikogeorgos, I. W. Fletcher, C. Boardman, P. Doyle, N. Ortuoste and G. J. Leggett, Biointerphases, 2010, 5, 6068.

90 S. Breakspear and J. R. Smith, J. Microsc., 2004, 215, 3439.

91 A. Ghorbal and A. B. Brahim, Polym. Test., 2013, 32, 11741180.

92 J. Fu, B. Li and Y. Han, J. Chem. Phys., 2005, 123, 64713.

93 T. Aoike, H. Uehara, T. Yamanobe and T. Komoto, Langmuir, 2001, 17, 2153-2159.

94 K. Tanaka, A. Takahara and T. Kajiyama, Macromolecules, 1997, 30, 6626-6632.

95 Z. Y. Zhang, I. W. Fletcher, C. R. Hurley, C. Boardman, P. Doyle and G. J. Leggett, J. Mater. Chem., 2010, 20, 85318538.
96 D. P. Chang, F. Guilak, G. D. Jay and S. Zauscher, J. Biomech., 2014, 47, 659-666.

97 Y. Yuan and A. M. Lenhoff, J. Colloid Interface Sci., 2003, 267, 352-359.

98 L. J. Landherr, C. Cohen and L. A. Archer, Langmuir, 2011, 27, 5944-5952.

99 A. Meurk, J. Yanez and L. Bergstrom, Powder Technol., 2001, 119, 241-249.

100 J. An, A. Dedinaite, A. Nilsson, J. Holgersson and P. M. Claesson, Biomacromolecules, 2014, 15, 1515-1525.

101 S. Park, K. D. Costa and G. A. Ateshian, J. Biomech., 2004, 37, 1679-1687.

102 K. Theander, R. J. Pugh and M. W. Rutland, J. Colloid Interface Sci., 2007, 313, 735-746.

103 N. Nordgren, P. Eronen, M. Osterberg, J. Laine and M. W. Rutland, Biomacromolecules, 2009, 10, 645-650.

104 A. Olszewska, J. J. Valle-Delgado, M. Nikinmaa, J. Laine and M. Osterberg, Nanoscale, 2013, 5, 11837-11844.

105 A. Olszewska, K. Junka, N. Nordgren, J. Laine, M. W. Rutland and M. Osterberg, Soft Matter, 2013, 9, 7448-7457.

106 J. Stiernstedt, N. Nordgren, L. Wagberg, H. Brumer 3rd, D. G. Gray and M. W. Rutland, J. Colloid Interface Sci., 2006, 303, 117-123.

107 J. Stiernstedt, H. Brumer 3rd, Q. Zhou, T. T. Teeri and M. W. Rutland, Biomacromolecules, 2006, 7, 2147-2153.

108 S. Zauscher and D. J. Klingenberg, Colloids Surf., A, 2001, 178, 213-229.

109 A. A. Feiler, J. Stiernstedt, K. Theander, P. Jenkins and M. W. Rutland, Langmuir, 2007, 23, 517-522.

110 D. Dean, J. Hemmer, A. Vertegel and M. Laberge, Materials, 2010, 3, 4668-4680.

111 S. N. Ramakrishna, M. Cirelli, E. S. Kooij, M. K. Gunnewiek and E. M. Benetti, Macromolecules, 2015, 48, 7106-7116.

112 T. Shoaib, J. Heintz, J. A. Lopez-Berganza, R. MuroBarrios, S. A. Egner and R. M. Espinosa-Marzal, Langmuir, 2018, 34, 756-765.

113 X. L. Zhang, F. Liu, W. Z. Wang, G. W. Yi and J. H. Jia, J. Adhes. Sci. Technol., 2013, 27, 2603-2614.

114 X. L. Zhang, X. Wang, W. Kong, G. W. Yi and J. H. Jia, Appl. Surf. Sci., 2011, 258, 113-119.

115 S. E. Majd, R. Kuijer, T. A. Schmidt and P. K. Sharma, Mater. Des., 2015, 83, 514-521.

116 X. L. Zhang, Y. J. Lu, E. Y. Liu, G. W. Yi and J. H. Jia, Colloids Surf., A, 2012, 401, 90-96.

117 X. L. Zhang, C. X. Wu, H. W. Che, J. X. Hou and J. H. Jia, Appl. Surf. Sci., 2014, 320, 328-333.

118 L. J. Landherr, C. Cohen, P. Agarwal and L. A. Archer, Langmuir, 2011, 27, 9387-9395.

119 L. Ma, A. Gaisinskaya-Kipnis, N. Kampf and J. Klein, Nat. Commun., 2015, 6, 6060.

120 A. Gaisinskaya, L. Ma, G. Silbert, R. Sorkin, O. Tairy, R. Goldberg, N. Kampf and J. Klein, Faraday Discuss., 2012, 156, 217-233.

121 S. Jahn and J. Klein, Macromolecules, 2015, 48, 5059-5075. 
122 M. T. Muller, X. P. Yan, S. W. Lee, S. S. Perry and N. D. Spencer, Macromolecules, 2005, 38, 5706-5713.

123 E. S. Dehghani, Y. H. Du, T. Zhang, S. N. Ramakrishna, N. D. Spencer, R. Jordan and E. M. Benetti, Macromolecules, 2017, 50, 2436-2446.

124 S. N. Ramakrishna, M. Cirelli, M. Divandari and E. M. Benetti, Langmuir, 2017, 33, 4164-4171.

125 E. S. Dehghani, S. N. Ramakrishna, N. D. Spencer and E. M. Benetti, Macromolecules, 2017, 50, 2932-2941.

126 N. Nordgren and M. W. Rutland, Nano Lett., 2009, 9, 2984-2990.

127 Y. Yu, B. D. Kieviet, F. Liu, I. Siretanu, E. Kutnyanszky, G. J. Vancso and S. de Beer, Soft Matter, 2015, 11, 8508-8516.

128 Y. L. Dong, Q. Y. Li and A. Martini, J. Vac. Sci. Technol., A, 2013, 31, 030801.

129 Q. Y. Li, Y. L. Dong, D. Perez, A. Martini and R. W. Carpick, Phys. Rev. Lett., 2011, 106, 126101.

130 H. M. Yoon, Y. Jung, S. C. Jun, S. Kondaraju and J. S. Lee, Nanoscale, 2015, 7, 6295-6303.

131 Y. Dong, Q. Li, J. Wu and A. Martini, Modell. Simul. Mater. Sci. Eng., 2011, 19, 065003.

132 J. G. Vilhena, C. Pimentel, P. Pedraz, F. Luo, P. A. Serena, C. M. Pina, E. Gnecco and R. Perez, ACS Nano, 2016, 10, 4288-4293.

133 P. Egberts, Z. J. Ye, X. Z. Liu, Y. L. Dong, A. Martini and R. W. Carpick, Phys. Rev. B: Condens. Matter Mater. Phys., 2013, 88, 035409.

134 A. Schlaich, J. Kappler and R. R. Netz, Nano Lett., 2017, 17, 5969-5976.
135 J. Han, J. P. Sun, S. Xu, D. Song, Y. Han, H. Zhu and L. Fang, Coatings, 2018, 8, 7.

136 R. T. Tong, G. Liu and T. X. Liu, Chin. J. Mech. Eng., 2013, 26, 1109-1117.

137 P. Z. Zhu, Y. Z. Hu, T. B. Ma and H. Wang, Tribol. Lett., 2011, 41, 41-46.

138 L. Dai, M. N. Satyanarayana, S. K. Sinha and V. B. C. Tan, Langmuir, 2011, 27, 14861-14867.

139 S. de Beer, E. Kutnyanszky, P. M. Schon, G. J. Vancso and M. H. Muser, Nat. Commun., 2014, 5, 3781.

140 M. K. Singh, C. J. Kang, P. Ilg, R. Crockett, M. Kroger and N. D. Spencer, Macromolecules, 2018, 51, 10174-10183.

141 S. M. T. Chan, C. P. Neu, G. DuRaine, K. Komvopoulos and A. H. Reddi, Osteoarthritis Cartilage, 2010, 18, 956963.

142 D. P. Chang, N. I. Abu-Lail, J. M. Coles, F. Guilak, G. D. Jay and S. Zauscher, Soft Matter, 2009, 5, 3438-3445.

143 M. Lekka, A. J. Kulik, S. Jeney, J. Raczkowska, J. Lekki, A. Budkowski and L. Forro, J. Chem. Phys., 2005, 123, 014702.

144 Z. Lv, J. H. Wang, G. P. Chen and L. H. Deng, J. Biol. Phys., 2011, 37, 417-427.

145 W. Tang and B. Bhushan, Colloids Surf., B, 2010, 76, 1-15.

146 B. Bhushan, S. Chen and S. R. Ge, Beilstein J. Nanotechnol., 2012, 3, 731-746.

147 M. Sadaie, N. Nishikawa, S. Ohnishi, K. Tamada, K. Yase and M. Hara, Colloids Surf., B, 2006, 51, 120-129.

148 F. Huang, K. C. Li and A. Kulachenko, J. Mater. Sci., 2009, 44, 3770-3776. 